\title{
Links between cardiovascular disease and osteoporosis in postmenopausal women: serum lipids or atherosclerosis per se?
}

\author{
Y. Z. Bagger $\cdot$ H. B. Rasmussen $\cdot$ P. Alexandersen • \\ T. Werge • C. Christiansen • L. B. Tankó • \\ PERF study group
}

Received: 18 August 2006 / Accepted: 9 October 2006 / Published online: 16 November 2006

(C) International Osteoporosis Foundation and National Osteoporosis Foundation 2006

\begin{abstract}
Introduction and hypothesis Epidemiological observations suggest links between osteoporosis and risk of acute cardiovascular events and vice versa. Whether the two clinical conditions are linked by common pathogenic factors or atherosclerosis per se remains incompletely understood. We investigated whether serum lipids and polymorphism in the ApoE gene modifying serum lipids could be a biological linkage.

Methods This was an observational study including 1176 elderly women $60-85$ years old. Women were genotyped for epsilon $(\varepsilon)$ allelic variants of the ApoE gene, and data concerning serum lipids (total cholesterol, triglycerides, HDL-C, LDL-C, apoA1, ApoB, Lp(a)), hip and spine $\mathrm{BMD}$, aorta calcification (AC), radiographic vertebral fracture and self-reported wrist and hip fractures, cardiovascular events together with a wide array of demographic and lifestyle characteristics were collected.

Results Presence of the ApoE $\varepsilon 4$ allele had a significant impact on serum lipid profile, yet no association with spine/ hip BMD or AC could be established. In multiple regression models, apoA1 was a significant independent contributor to the variation in AC. However, none of the
\end{abstract}

Y. Z. Bagger $(\bowtie) \cdot$ P. Alexandersen $\cdot$ C. Christiansen

L. B. Tankó

Center for Clinical and Basic Research $\mathrm{A} / \mathrm{S}$,

Ballerup Byvej 222,

DK-2750 Ballerup, Denmark

e-mail: yb@ccbr.dk

H. B. Rasmussen • T. Werge

Research Institute of Biological Psychiatry,

H:S Sct. Hans Hospital,

DK-4000 Roskilde, Denmark lipid components were independent contributors to the variation in spine or hip BMD. When comparing the women with or without vertebral fractures, serum triglycerides showed significant differences. This finding was however not applicable to hip or wrist fractures. After adjustment for age, severe AC score ( $\geq 6)$ and/or manifest cardiovascular disease increased the risk of hip but not vertebral or wrist fractures.

Conclusion The contribution of serum lipids to the modulators of BMD does not seem to be direct but rather indirect via promotion of atherosclerosis, which in turn can affect bone metabolism locally, especially when skeletal sites supplied by end-arteries are concerned. Further studies are needed to explore the genetic or environmental risk factors underlying the association of low triglyceride levels to vertebral fractures.

Keywords Aorta calcification - ApoE polymorphism . Cardiovascular events $\cdot$ Lipids · Osteoporosis

\section{Introduction}

Osteoporotic fractures and acute cardiovascular events remain the predominant contributors to morbidity and mortality among the elderly. Postmenopausal women with osteoporosis are at increased risk for acute cardiovascular events independent of their age and cardiovascular risk profile, and the increase in risk is proportional to the severity of osteoporosis at the time of the diagnosis [1]. Bone mineral density (BMD) at the hip is inversely correlated with the severity of aorta calcification (AC) and hence low hip BMD can be a surrogate marker of the atherosclerotic burden in elderly women [2]. Large cohort 
studies demonstrate that the rate of bone loss in metacarpals, proximal femur, and calcaneus is proportional to the progression of $\mathrm{AC}$ [3-5]. Finally, advanced $\mathrm{AC}$ is associated with increased risk of osteoporotic fractures [5, 6]. Collectively, numerous epidemiological observations document an overlap between the pathogenesis of the two diseases $[7,8]$.

An attractive concept is that cardiovascular disease and osteoporosis are linked by a common denominator acting in parallel on both vascular and bone cells $[9,10]$. Plausible candidates of such linking factors are serum lipids, which in addition to their established role in atherogenesis, were also shown to act on osteoblasts [11-13] and osteoclasts [14] under experimental conditions. Although some smaller cross-sectional studies found associations between lipid profile and BMD in humans [15-17], neither large population-based studies (e.g., NHANES III) nor prospective studies support these initial observations $[18,19]$. A possible explanation the discrepancies could be that the true driver of the association is obstructive vascular disease, meaning that the association of lipids to bone is not detectable before the severity of intraluminal lesions reaches a critical grade when blood supply to the anatomical region is considerably hampered. Until now there have been no epidemiological studies that investigated associations of lipids and lipoprotein components with bone independent of a direct surrogate of the atherosclerotic burden.

The aim of the present study was to test this hypothesis and investigate associations of a wide array of serum lipids and lipoproteins as well as of a genetic determinant of lipid profile with different surrogate measures of osteoporosis (BMD, fractures) and cardiovascular disease (AC, acute events) in 1176 Danish postmenopausal women providing full-set of data for such analyses.

\section{Subjects and methods}

\section{Study population}

The study population consisted of 1176 generally healthy postmenopausal women participating in the PERF study. Details on recruiting have been described elsewhere [20]. This subpopulation was selected based on those whose BMD and body composition were measured by the same DXA scanner. The population characteristics were not statistically significant from the total PERF population indicating that the results reported herein are generally applicable to elderly Caucasian women $60-85$ years old. None of the women received any approved antiresorptive treatment or any other drugs with known effect on bone mass for more than 1 year. Forty-two patients reporting ongoing treatment with lipid-lowering drugs was excluded when analyzing associations of allelic variation in the ApoE gene with lipid profile.

All women gave written their informed consent to participation and the study was carried out in compliance with the Helsinki Declaration II and the European Standards for Good Clinical Practice. The study protocol was approved by the local ethical committee.

Demographic characteristics, risk factors, and clinical events

Body weight and height were measured to the closest $0.1 \mathrm{~kg}$ and $0.1 \mathrm{~cm}$, respectively, in women wearing light indoor clothing and no shoes. Body mass index (BMI) was calculated as body weight (in $\mathrm{kg}$ ) divided by height squared (in $\mathrm{m}$ ). Information about age, years since menopause, smoking habits, regular alcohol and coffee consumption, weekly exercise, prevalent diabetes and cardiovascular diseases (stroke, coronary events, intermittent claudication, unstable angina) were collected during personal interview using a questionnaire [20].

\section{Bone mineral density and fat mass measurement}

Bone mineral density (BMD) of the lumbar spine L1-4 and total hip was measured by dual energy X-ray absorptiometry (DEXA) using a QDR-2000 scanner (Hologic Inc., Waltham, MA, USA). Daily phantom scans were performed each morning for proper quality control. Body composition also was measured by the same whole-body scanner. The two components measured were total body fat mass (TFM) in kilograms and total lean mass in kilograms [21]. Total body fat mass was divided into two depots: central fat mass (CFM, subcutaneous and visceral fat mass of the trunk) and peripheral fat mass (PFM, subcutaneous fat mass of the legs and arms). Body fat distribution was expressed by the CFM/PFM ratio, analogous to the wellknown waist-to-hip ratio [22].

Fracture diagnosis

Lateral X-rays of the thoracic and lumbar spine were performed using standard X-ray equipment. Vertebral deformities from T4 to L4 were assessed by digital measurements of vertebral deformations using the Image Pro Image Analyzer software (version 4.5 for windows, Media Cybernetics Inc., Silver Spring, MD, USA). The ratio of the anterior and posterior heights of each vertebral body was determined and a difference between the anterior and posterior edges exceeding $20 \%$ was considered as a radiographic vertebral fracture. Information on prevalent non-vertebral fractures (wrist, hip, humeral fracture, rib, ankle, and foot) was collected during 
personal interview and later verified by X-rays or hospital discharge summaries.

\section{Grading of aorta calcification}

AC was assessed on lateral radiographs. Briefly, calcified deposits in the lumbar aorta adjacent to each lumbar vertebra (L1-L4) were assessed separately for the anterior and posterior wall of the aorta using the midpoint of the inter-vertebral space as the boundaries. Each wall of each segment was graded for the presence of calcified deposits with a score from 0 to 3 (0: no deposits, 1: less than one-third of the aortic wall, 2: onethird to two-thirds of the aortic wall. 3: more than twothirds of the aortic wall covered with calcified deposits). The sum of the scores of individual aortic segments both for the anterior and posterior walls, termed as anteriorposterior severity score and was used to describe the overall severity of $\mathrm{AC}$ in the lumbar aorta. Maximum score possibly given was $4 \times 2 \times 3=24$. The same investigator, who was blinded for all other results of the individual participants, carried out the evaluations. Intrarater correlations between repeated measurements were in the range of $r=0.92-98 \quad(n=50)$.

\section{Laboratory parameters}

Blood samples were collected in the morning after fasting overnight $(>12 \mathrm{~h})$. Serum total cholesterol, triglyceride, and high-density lipoprotein (HDL) cholesterol were determined by enzymatic assay using a Vitros-250 (Johnson \& Johnson, Taastrup, Denmark). LDL cholesterol was calculated by Friedewald formula $(\mathrm{LDL}-\mathrm{C}=\mathrm{TC}-\mathrm{HDL}-\mathrm{C}-$ $0.45 \times \mathrm{TG})$. Lipoprotein(a), ApoA1, and ApoB were measured by the Cobas Mira (Hoffman-La Roche, Mannheim, Germany) automatic blood analyzer.

\section{Genotyping}

DNA was isolated from peripheral blood samples. ApoE epsilon 2, epsilon 3 and epsilon 4 alleles were determined by enzymatic amplification of genomic DNA followed by restriction enzyme treatment of the amplified products (Ossendorf and Prellwitz, Qiangen News 2000, issue1, 11-13). Briefly, amplified products were treated with AflII and HaeII, respectively, and subjected to electrophoretic separation in agarose gels. The sequences of the amplification primers were 5'-ACTGACCCCGGTGGCGGAGGA GACGCGTGC-3' and 5'-TGTTCCACCAGGGGCCC CAGGCGCTCGCGG-3'. One of these primers was deliberately designed with a mismatch (underlined) to create a non-polymorphic AflIII site permitting assessment of the efficiency of the digestion process.
Statistical analysis

Results are presented as mean $\pm \mathrm{SD}$, unless otherwise indicated. Confounders of hip BMD and AC were identified by univariate correlation analysis. We classified ApoE genotypes into two categories marked by absence or presence of the $\varepsilon 4$ allele. General linear models (GLM) tested the differences in selected dependent variables after adjustment for possible confounders. Similar approach was used to identify factors showing differences between women with or without fractures. Logistic regression models were established to assess the age-dependent and age-independent relative risk of wrist, hip, or vertebral fractures in women with advanced aorta calcification or cardiovascular disease. Statistical analyses were carried out using the SPSS data analysis software (version 12, SPSS Inc., Chicago, IL). All statistical tests were two-tailed and differences were considered significant if the p-value was less than 0.05 .

\section{Results}

Demographic characteristics

Demographic characteristics of the total population are shown in Table 1. Less than one fifth of the population with an average age of 69.3 years had one or more manifes-

Table 1 Demographic characteristics in the study population

Characteristics and equivalent values

\begin{tabular}{ll}
\hline No. of women & 1176 \\
Age (years) & $69.3 \pm 6.4$ \\
Age at menopause (years) & $48.7 \pm 5.0$ \\
BMI $\left(\mathrm{kg} / \mathrm{m}^{2}\right.$ ) & $26.4 \pm 4.4$ \\
Current smoking (\%) & $24.6 \%$ \\
Regular intake of alcohol (\%) & $55.2 \%$ \\
Serum total cholesterol (mmol/l) & $6.3 \pm 1.0$ \\
Serum triglyciders (mmol/l) & $1.4 \pm 0.7$ \\
Serum HDL (mmol/l) & $1.7 \pm 0.4$ \\
Serum LDL (mmol/1) & $4.0 \pm 0.9$ \\
Serum ApoA1 (mmol/1) & $203.9 \pm 37.1$ \\
Serum ApoB (mmol/1) & $120.3 \pm 24.5$ \\
Serum Lp(a) (mmol/l) & $31.9 \pm 34.6$ \\
ApoE $\varepsilon 4$ allele carrier & $367,31.2 \%$ \\
Total hip BMD (g/cm ${ }^{2}$ ) & $0.80 \pm 0.1$ \\
Lumbar spine BMD (g/cm ${ }^{2}$ ) & $0.92 \pm 0.2$ \\
Hip BMD T-score $\leq-2.5$ (no. of women, $\%)$ & $147,12.5 \%$ \\
Spine BMD T-score $\leq-2.5$ (no. of women, $\%)$ & $179,15.2 \%$ \\
Vertebral fracture (no. of women, \%) & $204,17.6 \%$ \\
Hip fracture (no. of women, $\%)$ & $16,1.4 \%$ \\
Aorta calcification & $3.0 \pm 3.8$ \\
Cardiovascular disease (no. of women, $\%$ ) & $50,4.3 \%$ \\
\hline
\end{tabular}

Data shown are mean \pm SD. 
tations of osteoporosis in form of a low BMD T-score equal or below -2.5 and/or a fracture. In terms of cardiovascular status, the mean severity score of $\mathrm{AC}$ was $3.0 \pm 3.8$ and the number of women with history of an acute cardiovascular event was $50(4.3 \%)$.

The ApoE genotype frequencies were $0.9 \%(\mathrm{n}=11)$ for ApoE $\varepsilon 2-2,12.7 \%(n=149)$ for ApoE $\varepsilon 2-3,2.5 \%(n=29)$ for ApoE ع2-4, 55.2\% (n=649) for ApoE ع3-3, 26.6\% $(n=313)$ for ApoE $\varepsilon 3-4$, and 2.1\% $(n=25)$ for ApoE $\varepsilon 4-4$, respectively. When classifying ApoE genotypes into absence of ApoE $\varepsilon 4$ allele and presence of ApoE $\varepsilon 4$ allele, $31.2 \%(n=367)$ of women were ApoE $\varepsilon 4$ allele carriers. These genotype proportions did not deviate significantly with those expected under conditions of Hardy-Weinberg equilibrium $\left(\mathrm{X}^{2}=4.55 ; \mathrm{df}=3 ; \mathrm{p}=0.21\right)$, and they were similar to those previously reported for Danish populations $[22,23]$.

Correlates of hip and spine BMD and AC

In univariate analyses, summarized in Table 2, AC showed direct correlation with age, CFM/PFM ratio, smoking, treated hypertension, and inverse association with physical exercise, regular alcohol consumption, BMI, and TFM\%. On the other hand, BMI, TFM $\%$ and CFM/PFM ratio were significant direct, whereas age, years since menopause, and smoking were inverse correlates of both spine and hip BMD. Treated hypertension was significantly associated with spine BMD, whereas the severity score of AC was a significant inverse correlate of hip BMD $(\mathrm{p}<0.05)$.
The independent role of the ApoE $\varepsilon 4$ allele

Results of comparing lipids and lipoproteins between women with or without the ApoE $\varepsilon 4$ allele are summarized in Table 3. After adjustment for confounders, women with an ApoE \&4 allele had significantly higher serum total cholesterol, triglycerides, LDL-C, and ApoB levels than women without this allele. Furthermore, presence of the ApoE $\varepsilon 4$ allele was associated with significantly lower HDL-C. In contrast, the differences in serum ApoA1 and Lp(a) did not reach statistical significance (Table 3). Finally, there were no significant differences in adjusted spine or hip BMD or the severity of AC ( $>0.05)$.

\section{Associations of lipids with BMD and AC}

In univariate analysis (Table 2), total cholesterol and triglycerides were common correlates of spine BMD, hip BMD, and AC. HDL-C and ApoA1 were common correlates of AC and hip BMD ( $p<0.05)$, whereas LDL-C, but not ApoB, was a common correlate of $\mathrm{AC}(\mathrm{p}<0.05)$.

To obtain insights into the direct contribution of lipids to the variation of hip and spine BMD and the severity of AC, we established multiple regression models. The independent contributors to the variation in these surrogate markers are indicated in Table 2. Lipids were not independent contributors to the variation in BMD when assessed in the presence of the severity of $\mathrm{AC}$ and other confounders. However, ApoA1 levels did seem to contribute to the variation in $\mathrm{AC}$.
Table 2 Independent correlates of aorta calcification, hip $\mathrm{BMD}$, and spine BMD (multiple regression models)

Excl.: excluded from the model due to strong co-linearity.

\begin{tabular}{llll}
\hline & Aorta Calcification & Hip BMD & Spine BMD \\
\hline Age & $0.35(\mathrm{p}<0.001)$ & $-0.23(\mathrm{p}<0.001)$ & $-0.03(\mathrm{p}=0.45)$ \\
YSM & $-0.06(\mathrm{p}=0.16)$ & $-0.06(\mathrm{p}=0.10)$ & $-0.08(\mathrm{p}=0.04)$ \\
BMI & $-0.09(\mathrm{p}=0.07)$ & $0.48(\mathrm{p}<0.001)$ & $0.45(\mathrm{p}<0.001)$ \\
TFM\% & $-0.04(\mathrm{p}=0.36)$ & $-0.10(\mathrm{p}=0.04)$ & $-0.21(\mathrm{p}<0.001)$ \\
CFM/PFM ratio & $0.08(\mathrm{p}=0.02)$ & $-0.004(\mathrm{p}=0.85)$ & $0.05(\mathrm{p}=0.21)$ \\
Smoking & $0.19(\mathrm{p}<0.001)$ & $-0.05(\mathrm{p}=0.10)$ & $-0.01(\mathrm{p}=0.66)$ \\
Exercise & $-0.03(\mathrm{p}=0.25)$ & $0.04(\mathrm{p}=0.17)$ & $0.04(\mathrm{p}=0.21)$ \\
Treated hypertension & $0.08(\mathrm{p}=0.006)$ & $0.006(\mathrm{p}=0.81)$ & $0.05(\mathrm{p}=0.09)$ \\
Alcohol consumption & $-0.08(\mathrm{p}=0.007)$ & $0.06(\mathrm{p}=0.04)$ & $0.07(\mathrm{p}=0.02)$ \\
ApoE $\varepsilon 4$ & $-0.009(\mathrm{p}=0.77)$ & $0.01(\mathrm{p}=0.76)$ & $-0.03(\mathrm{p}=0.22)$ \\
Triglyceride & $0.02(\mathrm{p}=0.63)$ & $0.03(\mathrm{p}=0.43)$ & $0.04(\mathrm{p}=0.32)$ \\
HDL-C & $0.05(\mathrm{p}=0.48)$ & $-0.06(\mathrm{p}=0.29)$ & $0.02(\mathrm{p}=0.74)$ \\
LDL-C & Excl. & $0.02(\mathrm{p}=0.73)$ & $-0.10(\mathrm{p}=0.10)$ \\
Total cholesterol & $0.05(\mathrm{p}=0.43)$ & Excl. & Excl. \\
ApoA1 & $-0.10(\mathrm{p}=0.04)$ & $0.05(\mathrm{p}=0.36)$ & $0.05(\mathrm{p}=0.39)$ \\
ApoB & $0.10(\mathrm{p}=0.14)$ & $-0.03(\mathrm{p}=0.60)$ & $0.10(\mathrm{p}=0.85)$ \\
Aorta Calcification & & $-0.09(\mathrm{p}=0.002)$ & $0.04(\mathrm{p}=0.20)$ \\
& $\mathrm{R}=0.44, \mathrm{SEE}=3.32$ & $\mathrm{R}=0.55, \mathrm{SEE}=0.09$ & $\mathrm{R}=0.34, \mathrm{SEE}=0.13$ \\
& $\mathrm{P}<0.001$ & $\mathrm{P}<0.001$ & $\mathrm{P}<0.001$ \\
\hline
\end{tabular}


Table 3 Lipid and bone profile in women with or without an ApoE epsilon 4 allele

\begin{tabular}{llll}
\hline & $\begin{array}{l}\text { Absence } \\
\text { of ApoE } \\
\text { epsilon } 4 \\
\text { allele } \\
(\mathrm{n}=809)\end{array}$ & $\begin{array}{l}\text { Presence } \\
\text { of ApoE } \\
\text { epsilon } 4 \\
\text { allele } \\
(\mathrm{n}=367)\end{array}$ & P value \\
& $6.24 \pm 0.03$ & $6.51 \pm 0.05$ & $<0.001$ \\
\hline Serum total cholersterol* & & & \\
$\quad(\mathrm{mmol} / \mathrm{l})$ & $1.36 \pm 0.02$ & $1.48 \pm 0.03$ & 0.005 \\
Serum triglycides* $(\mathrm{mmol} / \mathrm{l})$ & $1.65 \pm 0.02$ & 0.006 \\
Serum HDL* $(\mathrm{mmol} / \mathrm{l})$ & $1.72 \pm 0.01$ & $4.19 \pm 0.05$ & $<0.001$ \\
Serum LDL* $(\mathrm{mmol} / \mathrm{l})$ & $3.91 \pm 0.03$ & $201.3 \pm 2.0$ & 0.06 \\
Serum ApoA1* $(\mathrm{mmol} / \mathrm{l})$ & $205.8 \pm 1.3$ & $127.2 \pm 1.3$ & $<0.001$ \\
Serum ApoB* $(\mathrm{mmol} / \mathrm{l})$ & $117.3 \pm 0.8$ & $29.2 \pm 2.0$ & 0.24 \\
Serum Lp(a)* $(\mathrm{mmol} / \mathrm{l})$ & $31.9 \pm 1.3$ & $0.804 \pm 0.005$ & 0.89 \\
Total hip BMD $\left(\mathrm{g} / \mathrm{cm}^{2}\right)^{*}$ & $0.803 \pm 0.004$ & 0.32 \\
Spine BMD $\left(\mathrm{g} / \mathrm{cm}^{2}\right)^{*}$ & $0.924 \pm 0.005$ & $0.915 \pm 0.008$ & 0.32 \\
Hip fracture & $1.7 \%$ & $0.5 \%$ & 0.17 \\
Vertebral fractures & $16.4 \%$ & $19.3 \%$ & 0.24 \\
Severity score of AC** & $2.81 \pm 0.14$ & $2.97 \pm 0.21$ & 0.13 \\
Cardiovascular disease & $4.0 \%$ & $4.9 \%$ & 0.44 \\
\hline
\end{tabular}

Values are mean \pm SEM.

*Adjustment for age, years since menopause, BMI, TFM\%, CFM/ PFM ratio, current smoking, physical exercise, and regular alcohol consumption.

**Adjustment for the aforementioned confounders + treated hypertension.

Table 4 Characteristics of women with or without at least one vertebral fracture

\begin{tabular}{llll}
\hline & $\begin{array}{l}\text { Without } \\
\text { vertebral } \\
\text { fracture }\end{array}$ & $\begin{array}{l}\text { With } \\
\text { vertebral } \\
\text { fracture }\end{array}$ & p-value \\
& $68.9 \pm 6.4$ & $71.3 \pm 6.4$ & $<0.001$ \\
\hline Age & $12.7 \pm 8.4$ & $15.5 \pm 8.6$ & $<0.001$ \\
YSM & $26.4 \pm 4.4$ & $26.3 \pm 4.4$ & NS \\
BMI & $41.3 \pm 7.6$ & $40.7 \pm 7.8$ & NS \\
TFM\% & $0.88 \pm 0.23$ & $0.90 \pm 0.24$ & NS \\
CFM/PFM ratio & $73.8 \%$ & $67.6 \%$ & 0.08 \\
Regular exercise & $30.5 \%$ & $34.8 \%$ & NS \\
ApoE $\varepsilon 4$ allele + & $6.32 \pm 1.00$ & $6.31 \pm 1.04$ & $\mathrm{NS}$ \\
Total cholesterol & $1.42 \pm 0.69$ & $1.31 \pm 0.54$ & 0.02 \\
Triglycerides & $1.68 \pm 0.43$ & $1.71 \pm 0.39$ & $\mathrm{NS}$ \\
HDL-C & $3.98 \pm 0.93$ & $4.01 \pm 0.96$ & $\mathrm{NS}$ \\
LDL-C & $203.8 \pm 37.8$ & $204.6 \pm 33.4$ & $\mathrm{NS}$ \\
ApoA1 & $120.6 \pm 24.5$ & $119.1 \pm 24.5$ & $\mathrm{NS}$ \\
ApoB & $31.7 \pm 34.9$ & $32.5 \pm 33.5$ & $\mathrm{NS}$ \\
Lp(a) & $0.93 \pm 0.15$ & $0.87 \pm 0.14$ & $<0.001$ \\
Spine BMD & $0.82 \pm 0.11$ & $0.75 \pm 0.11$ & $<0.001$ \\
Hip BMD & $1.0 \%$ & $2.9 \%$ & 0.04 \\
Hip fracture & $11.4 \%$ & $14.6 \%$ & 0.04 \\
Wrist fracture & $2.8 \pm 3.7$ & $3.5 \pm 3.4$ & 0.03 \\
AC score & $3.6 \%$ & $7.4 \%$ & 0.02 \\
CVD & & & \\
\hline
\end{tabular}

There were no significant differences in the frequency of smoking, regular alcohol, coffee, milk or seasonal vitamin D consumption, type 2 diabetes, treated hyperlipidemia or treated hypertension
Comparison of women with or without fractures

There were altogether 293 subjects who had an osteoporotic fracture after the menopause (i.e., prevalent hip, vertebral, or wrist fracture). Of the 204 women with at least one vertebral fracture, 33 also had a history of wrist fracture and 16 reported a history of hip fracture. Prevalence of nonvertebral fractures was significantly higher in women with compared with those without vertebral fracture(s). Characteristics of women with or without vertebral fractures are summarized in Table 4. Women with fractures were on average older and had a tendency for being physically less active. However, no significant differences in BMI, body adiposity, or body fat distribution were apparent. In addition, no significant differences in a wide array of lifestyle factors were apparent.

When comparing the lipid profile of women with or without osteoporotic fractures after adjustment for potential confounders (i.e., age, years since menopause, BMI, TFM $\%$, CFM/PFM ratio, smoking, ApoE $\varepsilon 4$ allele, physical exercise, alcohol consumption, and severity score of $\mathrm{AC}$ ), only triglycerides showed significant differences between the two groups $(\mathrm{p}<0.05)$. Thus, triglycerides in women with and without fractures were $1.31 \pm 0.04 \mathrm{mmol} / \mathrm{l}$ and $1.42 \pm$ $0.03 \mathrm{mmol} / \mathrm{l}$, respectively. When analyzing fracture types separately, the difference between women with or without hip or wrist fractures were not statistically significant.

The relative risk of hip or vertebral fracture in women with severe AC or CVD

To assess the implications of atherosclerotic vascular disease for osteoporotic fracture risk, we calculated the odds ratio of the different types of fractures in women with severe AC $(\geq 6)$ and/or manifest CVD. As indicated in Table 5, after adjustment for age, the risk of hip fractures but not wrist or vertebral fractures was increased in subjects with advanced vascular disease.

Table 5 Relative risk of different types of osteoporotic fractures in women with severe aorta calcification (score $\geq 6$ ) and/or manifest cardiovascular disease $(n=282)$

\begin{tabular}{llll}
\hline & & $\begin{array}{l}\text { Odds ratio } \\
(95 \% \mathrm{CI})\end{array}$ & p-value \\
\hline Hip fracture & Crude & $4.4(1.6-12.9)$ & 0.001 \\
$\mathrm{~N}=16$ & Age-adjusted & $3.0(1.1-8.8)$ & 0.04 \\
Vertebral fracture & Crude & $1.2(0.9-1.7)$ & 0.27 \\
$\mathrm{~N}=204$ & Age-adjusted & $1.0(0.7-1.4)$ & 0.93 \\
$\mathrm{~W} r i s t$ fracture & Crude & $1.4(1.0-2.1)$ & 0.06 \\
$\mathrm{~N}=145$ & Age-adjusted & $1.2(0.8-1.7)$ & 0.46 \\
\hline
\end{tabular}




\section{Discussion}

In the present study, we investigated whether serum lipid and lipoproteins represent a biological linkage between osteoporosis and cardiovascular disease. The main findings were as follows: 1) neither allelic polymorphism in the ApoE gene nor serum lipids per se were independent correlates of BMD at any skeletal site, 2) ApoA1 levels were independent correlates of $\mathrm{AC}$, which seem to play a role in the determination of hip BMD, 3) decreased triglycerides were independently associated with vertebral fractures, but not with non-vertebral fractures (hip and wrist), and 4) advanced vascular disease was a significant contributor to risk of hip fractures, but not to vertebral or wrist fractures. These observations suggest that the role of lipids as linking factors between cardiovascular disease and osteoporosis is indirect via promotion of atherogenesis, which in turn can contribute to bone loss, especially at skeletal sites with end-arterial blood supply. Antiatherogenic measures can also be expected to contribute to decreasing the burden of hip fractures among the elderly.

Univariate analysis pointed out several common correlates of BMD and atherosclerosis. Of these factors, aging, the duration of the menopause, BMI, and TFM $\%$ seemed to have reciprocal impact on BMD and AC. In multiple regression models, whereas BMI continued to correlate directly, TFM $\%$ became an inverse correlate of BMD. Accordingly, while weight-bearing may have a favourable impact on bone metabolism, adipose tissue accumulation exert an adverse influence. These findings corroborate the recent findings of $\mathrm{Hsu} \mathrm{YH}$ et al. [24]. Although traditional views considered obesity a protective factor due to endogenous estrogen production, these more recent findings emphasize that other factors deriving from adipose tissue, e.g., adipokines, inflammatory cytokines, and perhaps even lipids/lipoprotein metabolism are also to be taken into account. In addition, these findings also draw attention to the need of proper adjustment for overall adiposity and body fat distribution when addressing the direct implication of selected potential mediators.

To address whether serum lipids represent a biological linkage between osteoporosis and vascular disease, one approach is to investigate whether polymorphism in a gene with known modulator effects on serum lipid profile is associated with variation in the severity of $\mathrm{AC}$ and BMD. Similar to previous observations $[25,26]$, we found that presence of the $\varepsilon 4$ variant allele in the ApoE gene was independently associated with higher levels of total cholesterol, LDL-C and ApoB. In addition, we also observed subtle, yet statistically significant differences in serum triglycerides and HDL-C levels. Nevertheless, we were not able to document significant associations of the $\varepsilon 4$ allele with either BMD or AC score. In male or mixed populations, the $\varepsilon 4$ allele was reported as a risk factor for atherosclerosis [27, 28], suggesting that the association might be gender-specific for males. Total cholesterol and LDL-C are important predictors of cardiovascular risk in men, but less so in postmenopausal women in whom triglycerides and HDL-C carry greater importance [29, 30]. Another explanation to the apparent discrepancies could be geographic variation in the relative importance of allelic variation in the ApoE gene. In support, a study in Finnish men reported higher importance of the $\varepsilon 3$ allele for atherogenesis compared with the $\varepsilon 4$ allele [27]. Similar discrepancies exist concerning the relative importance of this gene polymorphism for the determination of bone mass. Although there are reports suggesting that presence of the $\varepsilon 4$ allele is a contributor to accelerated bone loss and/ or increased risk of osteoporotic fractures [31,32], not all studies confirm this finding, particularly those undertaken in women [33-35]. Collectively, ApoE polymorphism with primary influence on total cholesterol, LDL-C, and apoB levels does not seem to provide a major biological linkage between the pathogenesis of osteoporosis and CVD.

Experimental observations proposed LDL-C and HDL-C as common modulators of bone cells and vascular smooth muscle cells [11-14]. On the clinical side, smaller crosssectional studies found associations between lipid profile and BMD [15-17]. However, none of these studies adjusted systematically for a direct DEXA measure of body fat distribution and/or smoking habits, which are important confounders of lipid profile. Our multiple regression analyses could not reveal significant independent associations of any of the considered lipid and lipoprotein components with hip or spine BMD. These findings are in line with the largest population-based observational study, the NHAEMS III survey including more than 13,000 subjects [19]. In further support, a recent longitudinal analysis of results from the Framingham study cohort reported insignificant direct influence of serum lipids for changes of BMD during a 25-year observational period [18]. Collectively, these observations do not seem to nurture the concept that serum lipids and lipoproteins exert clinically detectable direct effects on bone mineralization in elderly women.

To further assess the potential implications of serum lipids and lipoproteins for osteoporosis, we also compared the profile of postmenopausal women with or without osteoporotic fractures. Interestingly, serum triglycerides were significantly lower in women with at least one vertebral fracture compared with those with no fractures, while no differences were seen between those with or without an osteoporotic non-vertebral fracture (i.e., hip or wrist fracture). This finding is similar to that reported by Yamaguchi et al. [15]. Since triglycerides were not associated with spine BMD, their direct contribution to 
fracture risk, if any, is via modulation of bone quality or bone strength. Given the cross-sectional design of this study, we cannot exclude the possibility that a confounder or confounders of triglyceride levels, which was not considered herein is the actual modulator of bone fragility and hence fracture risk.

Important finding of the multiple regression analysis was that the severity of $\mathrm{AC}$ was an independent correlate of hip BMD with no simultaneous independent contribution of lipids. This observation provides evidence that obstructive vascular disease rather than lipids/lipoproteins per se that drive bone loss from the hip. In a previous study, we showed that women with intermittent claudication have not only severe AC, but also very low BMD [2]. The direct contribution of atherosclerosis is also supported by the observation showing that asymmetric vascular disease is accompanied by asymmetric demineralization of this skeletal site [36]. Moreover, studies show that the rate of demineralization at the hip is significantly associated with the rate of atherogenesis [5] and even future risk of cardiovascular events [37]. On the other hand, we recently demonstrated in a large population-based cohort of postmenopausal women that accelerated atherogenesis is an independent predictor of hip fracture risk. Histological investigations of explants from elderly patients with femoral neck fractures indicate that both the larger (a. iliaca ext and a. femoris profunda) and the small vessels in the ligamentum teres supplying this skeletal site are frequently obstructed by atheromatous lesions [38, 39]. These observations provide strong support for the important role of atherosclerosis for the pathogenesis of osteoporosis in the proximal femur.

In summary, the results of the present observational study provide further evidence for the independent association of peripheral vascular disease with osteoporosis in the proximal femur. Since the association of lipids and lipoproteins to $\mathrm{BMD}$ and non-vertebral fractures is not independent of the severity of $\mathrm{AC}$, it seems unlikely that these metabolites exert direct and clinically significant effects on bone turnover in postmenopausal women. Their contribution is via promotion of atherogenesis, in which regard ApoA1 levels seem to take a leading role. The remaining issue to be clarified is which genetic or environmental factors underlie the association of low triglycerides levels to vertebral fractures.

\section{References}

1. Tanko LB, Christiansen C, Cox DA, Geiger MJ, McNabb MA, Cummings SR (2005) Relationship between osteoporosis and cardiovascular disease in postmenopausal women. J Bone Miner Res 20(11):1912-1920, Epub, 18 Jul, Nov 2005
2. Tanko LB, Bagger YZ, Christiansen C (2003) Low bone mineral density in the hip as a marker of advanced atherosclerosis in elderly women. Calcif Tissue Int 73(1):15-20, Jul

3. Kiel DP, Kauppila LI, Cupples LA, Hannan MT, O’Donnell CJ, Wilson PW (2001) Bone loss and the progression of abdominal aortic calcification over a 25 year period: the Framingham Heart Study. Calcif Tissue Int 68(5):271-276, May

4. Hak AE, Pols HA, van Hemert AM, Hofman A, Witteman JC (2000) Progression of aortic calcification is associated with metacarpal bone loss during menopause: a population-based longitudinal study. Arterioscler Thromb Vasc Biol 20(8):1926-1931, Aug

5. Bagger YZ, Tanko LB, Alexandersen P, Qin G, Christiansen C (2006) Radiographic measure of aorta calcification is a sitespecific predictor of bone loss and fracture risk at the hip. J Intern Med 259(6):598-605, Jun

6. Schulz E, Arfai K, Liu X, Sayre J, Gilsanz V (2004) Aortic calcification and the risk of osteoporosis and fractures. J Clin Endocrinol Metab 89(9):4246-4253, Sep

7. Tanko LB, Bagger YZ, Nielsen SB, Christiansen C (2003) Does serum cholesterol contribute to vertebral bone loss in postmenopausal women? Bone 32(1):8-14, Jan

8. Frye MA, Melton LJ, III, Bryant SC et al (1992) Osteoporosis and calcification of the aorta. Bone Miner 19(2):185-194, Nov

9. Mody N, Tintut Y, Radcliff K, Demer LL (2003) Vascular calcification and its relation to bone calcification: possible underlying mechanisms. J Nucl Cardiol 10(2):177-183, Mar-Apr

10. Baldini V, Mastropasqua M, Francucci CM, D'Erasmo E (2005) Cardiovascular disease and osteoporosis. J Endocrinol Invest 28 (10 Suppl):69-72

11. Parhami F, Basseri B, Hwang J, Tintut Y, Demer LL (2002) Highdensity lipoprotein regulates calcification of vascular cells. Circ Res 91(7):570-576, 4 Oct

12. Parhami F, Garfinkel A, Demer LL (2000) Role of lipids in osteoporosis. Arterioscler Thromb Vasc Biol 20(11):2346-2348, Nov

13. Parhami F, Morrow AD, Balucan J et al (1997) Lipid oxidation products have opposite effects on calcifying vascular cell and bone cell differentiation. A possible explanation for the paradox of arterial calcification in osteoporotic patients. Arterioscler Thromb Vasc Biol 17(4):680-687, Apr

14. Tintut Y, Morony S, Demer LL (2004) Hyperlipidemia promotes osteoclastic potential of bone marrow cells ex vivo. Arterioscler Thromb Vasc Biol 24(2):e6-e10, Epub 2003, Dec 11, Feb

15. Yamaguchi T, Sugimoto T, Yano S et al (2002) Plasma lipids and osteoporosis in postmenopausal women. Endocr J 49(2):211-217, Apr

16. Adami S, Braga V, Zamboni M et al (2004) Relationship between lipids and bone mass in 2 cohorts of healthy women and men. Calcif Tissue Int 74(2):136-142, Epub 2003, 15 Dec, Feb

17. Brownbill RA, Ilich JZ (2006) Lipid profile and bone paradox: higher serum lipids are associated with higher bone mineral density in postmenopausal women. J Women's Health (Larchmt) 15(3):261-270, Apr

18. Samelson EJ, Cupples LA, Hannan MT et al (2004) Long-term effects of serum cholesterol on bone mineral density in women and men: the Framingham Osteoporosis Study. Bone 34(3):557561, Mar

19. Solomon DH, Avorn J, Canning CF, Wang PS (2005) Lipid levels and bone mineral density. Am J Med 118(12):1414, Dec

20. Bagger YZ, Tanko LB, Alexandersen P, Hansen HB, Qin G, Christiansen C (2006) The long-term predictive value of bone mineral density measurements for fracture risk is independent of the site of measurement and the age at diagnosis: results from the Prospective Epidemiological Risk Factors study. Osteoporos Int 17(3):471-477, Epub, 28 Oct 2005

21. Tanko LB, Bagger YZ, Alexandersen P, Larsen PJ, Christiansen C (2003) Central and peripheral fat mass have contrasting effect on 
the progression of aortic calcification in postmenopausal women. Eur Heart J 24(16):1531-1537, Aug

22. Mortensen EL, Hogh P (2001) A gender difference in the association between APOE genotype and age-related cognitive decline. Neurology 57:89-95

23. Gerdes LU, Vestergaard P, Hermann AP, Mosekilde L (2001) Regional and hormone-dependent effects of apolipoprotein E genotype on changes in bone mineral in perimenopausal women. J Bone Miner Res 16:1906-1916

24. Hsu YH, Venners SA, Terwedow HA et al (2006) Relation of body composition, fat mass, and serum lipids to osteoporotic fractures and bone mineral density in Chinese men and women. Am J Clin Nutr 83(1):146-154, Jan

25. Gregg RE, Zech LA, Schaefer EJ, Stark D, Wilson D, Brewer HB $\mathrm{Jr}$ (1986) Abnormal in vivo metabolism of apolipoprotein E4 in humans. J Clin Invest 78(3):815-821, Sep

26. Havel RJ, Chao Y, Windler EE, Kotite L, Guo LS (1980) Isoprotein specificity in the hepatic uptake of apolipoprotein $\mathrm{E}$ and the pathogenesis of familial dysbetalipoproteinemia. Proc Natl Acad Sci USA 77(7):4349-4353, Jul

27. Bleil ME, Ferrell RE, Sutton-Tyrrell K, Muldoon MF, Manuck SB (2006) Apolipoprotein E polymorphism and preclinical carotid artery disease in untreated hypertensive men. Eur J Cardiovasc Prev Rehabil 13(1):98-100, Feb

28. Lehtinen S, Lehtimaki T, Sisto T et al (1995) Apolipoprotein E polymorphism, serum lipids, myocardial infarction and severity of angiographically verified coronary artery disease in men and women. Atherosclerosis 114(1):83-91, 7 Apr

29. Bass KM, Newschaffer CJ, Klag MJ, Bush TL (1993) Plasma lipoprotein levels as predictors of cardiovascular death in women. Arch Intern Med 153(19):2209-2216, 11 Oct

30. Howard BV, Ruotolo G, Robbins DC (2003) Obesity and dyslipidemia. Endocrinol Metab Clin N Am 32(4):855-867, Dec

31. Cauley JA, Zmuda JM, Yaffe K et al (1999) Apolipoprotein E polymorphism: A new genetic marker of hip fracture risk-The
Study of Osteoporotic Fractures. J.Bone Miner Res 14(7):11751181, Jul

32. Salamone LM, Cauley JA, Zmuda J et al (2000) Apolipoprotein E gene polymorphism and bone loss: estrogen status modifies the influence of apolipoprotein $\mathrm{E}$ on bone loss. J Bone Miner Res 15:308-314

33. Sennels HP, Sand JC, Madsen B, Lauritzen JB, Fenger M, Jorgensen HL (2003) Association between polymorphisms of apolipoprotein E, bone mineral density of the lower forearm, quantitative ultrasound of the calcaneus and osteoporotic fractures in postmenopausal women with hip or lower forearm fracture. Scand J Clin Lab Invest 63(4):247-258

34. Efstathiadou Z, Koukoulis G, Stakias N, Challa A, Tsatsoulis A (2004) Apolipoprotein E polymorphism is not associated with spinal bone mineral density in peri- and postmenopausal Greek women. Maturitas 48(3):259-264, 15 Jul

35. Schoofs MW, van der KM, Hofman A et al (2004) ApoE gene polymorphisms, BMD, and fracture risk in elderly men and women: the Rotterdam study. J Bone Miner Res 19(9):14901496, Epub (2004) 21 Jun, Sep

36. Laroche M, Pouilles JM, Ribot C et al (1994) Comparison of the bone mineral content of the lower limbs in men with ischaemic atherosclerotic disease. Clin Rheumatol 13(4): 611-614, Dec

37. Kado DM, Browner WS, Blackwell T, Gore R, Cummings SR (2000) Rate of bone loss is associated with mortality in older women: a prospective study. J Bone Miner Res 15(10):1974 1980, Oct

38. Parhami F, Mody N, Gharavi N, Ballard AJ, Tintut Y, Demer LL (2002) Role of the cholesterol biosynthetic pathway in osteoblastic differentiation of marrow stromal cells. J Bone Miner Res 17 (11):1997-2003, Nov

39. Meier CR, Schlienger RG, Kraenzlin ME, Schlegel B, Jick H (2000) HMG-CoA reductase inhibitors and the risk of fractures. JAMA 283(24):3205-3210, 28 Jun 